\title{
INTEGRATING SATELLITE DIGITAL RADIO BROADCASTING (S-DB), TERRESTRIAL CELLULAR TECHNOLOGY AND EGNOS SATELLITE NAVIGATION \\ The RELY project
}

\author{
O. Courseille*, P. Poiré *, M.C. Durand **,M. Mazzella*** \\ Alcatel Space ${ }^{*}$ Toulouse, ${ }^{* *}$ Cannes, ${ }^{* * *}$ Nanterre
}

\begin{abstract}
This paper presents the IST RELY project which aims at Integrating Satellite Digital Radio Broadcasting (S-DB), terrestrial cellular technology and EGNOS satellite navigation
\end{abstract}

Key words: EGNOS satellite navigation, cellular technology, satellite radio broadcasting

\section{INTRODUCTION}

The RELY project is an European Commission -IST project that has the following objectives :

- To demonstrate the provision of multicast push-and-store services to invehicle mobile terminals using an hybrid satellite-terrestrial broadcasting system on a European dimension.

- To provide EGNOS service in an innovative, cost effective and global manner using standard mass marketed satellite Digital Broadcasting (SDB) features and hardware. The goal is to contribute to the development and the operations of the "integrity" market and to explore new applications. RELY will also facilitate the development of EGNOS outside Europe.

- A set of services using the different satellite technologies will be defined and implemented.

RELY is a 27 months project (April 2002 till June 2004). 
The following companies are involved in the project:

- Alcatel Space

- Webraska Mobile Technologies SA

- Fraunhofer-Gesellschaft zur Förderung der angewandten Forschung e.V.

- Volvo Teknisk Utveckling Aktiebolag

- Borg Instruments AG

- Worldspace France

- Alcatel Mobicom Europe SA

- Intellicast SA

- Daimler Chrysler AG

- Bayerische Motorenwerke AG

\section{RELY SYSTEM ARCHITECTURE}

A segment description of a satellite digital radio system is given in the following figure :

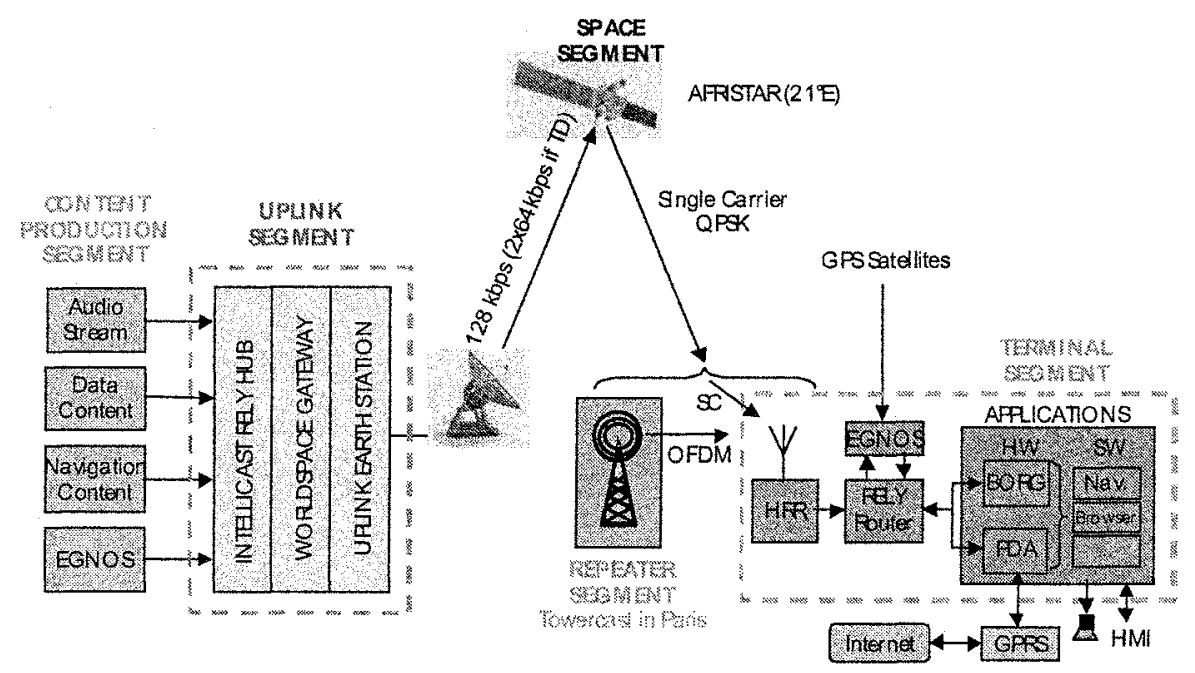

Fig.1: RELY Segments Architecture 


\subsection{Content Production Segment}

The Content Production Segment (CP-SEG) is composed of several service providers facilities connected to the up-link station.

The CP-SEG mission is :

- To get or create the content,

- To label batch or stream content to enable intelligent filtering in the terminal,

- To transmit the labelled content to the Up-Link Segment (UL-SEG).

- To negotiate bandwidth.

The CP-SEG is interfaced with content providers on one side (although this interface is not part of RELY focus), and with the UL-SEG on the other side. The CP-SEG constraints are the following.

- Adapted to the small displays available in vehicle or on handheld devices,

- Easy to access for a driving user.

The roles of the content production segment are to produce contents characterised by meta-data and to upload them to the uplink segment for subsequent broadcast over the satellite.

We can distinguish two types of contents :

- the real-time streams: in that category, we find the real-time audio stream and the EGNOS stream. These subsystems collocated with the uplink segment interface with the uplink segment at LAN level

- the file-based content: all other contents such as traffic information, pushed songs/news, points of interests,...These subsystems interface with the uplink segment using Internet.

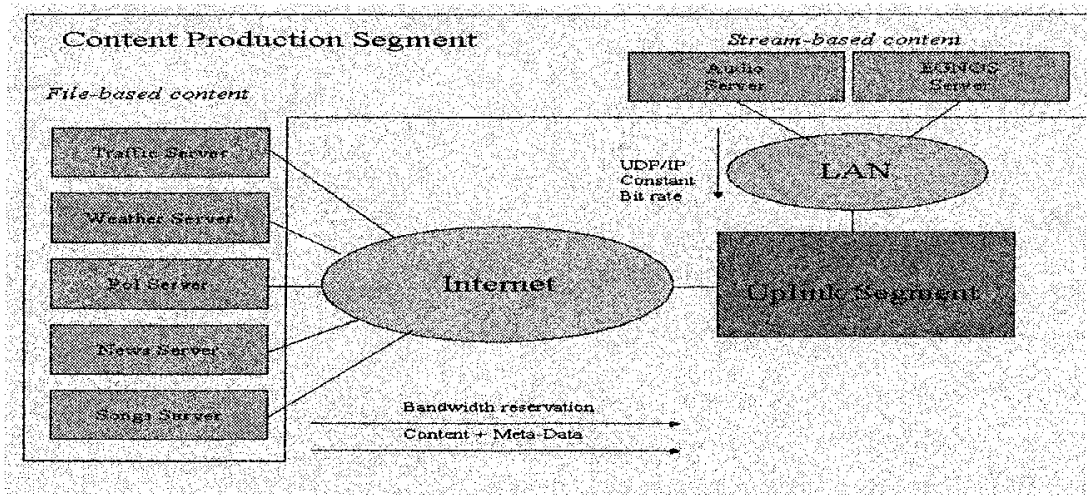

Fig. 2 : Content Production Segment Architecture 


\subsubsection{Audio Service Production Subsystem}

The audio production segment is collocated with the uplink segment in Erlangen. The architecture of the audio subsystem is illustrated here below:

Figure 3 - Audio Service Production Subsystem

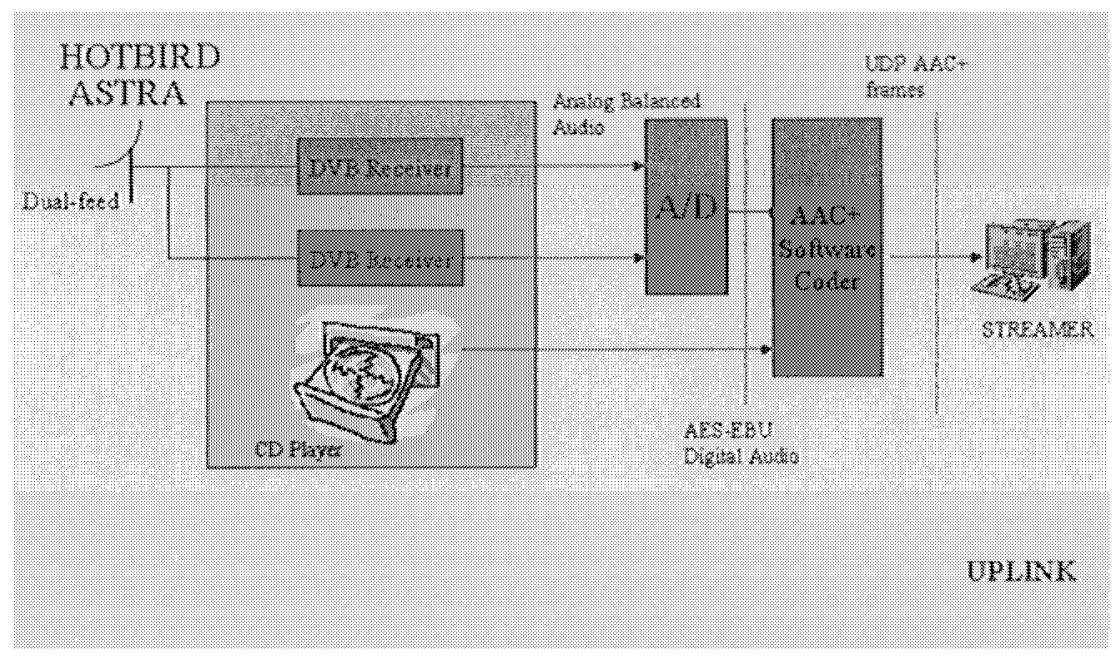

This subsystem consists in a 1.2 meter dual-feed Ku-band dish and two professional Tandberg TT1220 DVB receivers. One receiver is tuned to the TPS bouquet (HotBird) and decrypts the main French radios using a standard Conditional Access Module (ViaAccess) and a smart card. The second receiver is tuned to the German radios, available in clear over Astra. This receiver decodes the MPEG encoded audio and outputs a balanced analogue audio signal. A manual patch allows to select the signal to be broadcast. The selected signal goes through an analogue-to-digital converter to generate an AES-EBU digital uncompressed audio signal.

A CT-AAC+ audio software codec from Coding Technologies (G) receives the AES-EBU digital uncompressed signal from an audio PCI sound board. It performs on-the-fly encoding of the audio into CT-AAC+ frames.

The bandwidth reserved for the audio stream is constant and equal to 40 kbps in order to broadcast stereo programs. UDP is used as the transmission protocol from the audio server to the RELY streamer (Uplink Segment) over a standard LAN. Only the original CT-AAC+ frames (encapsulated in UDP) are broadcast.

A professional CD player is also available for back-up purposes as well as reference audio quality measurement. 


\section{$2.2 \quad$ Up-Link Segment}

The Up-Link Segment (UL-SEG) is composed of one station located in Erlangen. The UL-SEG mission is :

- To receive the content from several service providers,

- To encrypt the content of pay services (not implemented in RELY),

- To perform reliable transport layer encoding in order to ensure robustness to long reception disruptions caused by mobile propagation conditions,

- To achieve data compression,

- To implement a transmission strategy adapted to each category of content (example: for instance an information updated every 12 hours shall be retransmitted in the meantime in order to prevent users whose vehicle was not able to receive one occurrence from having to wait 12 more hours to get the information),

- To create a program channel or announcement channel,

- To multiplex the data received from several service providers,

- To implement time-diversity (i.e. the content is broadcast twice with $4 \mathrm{~s}$ delay in order to withstand short interruptions at the terminal level when driving under a bridge on behind buildings).

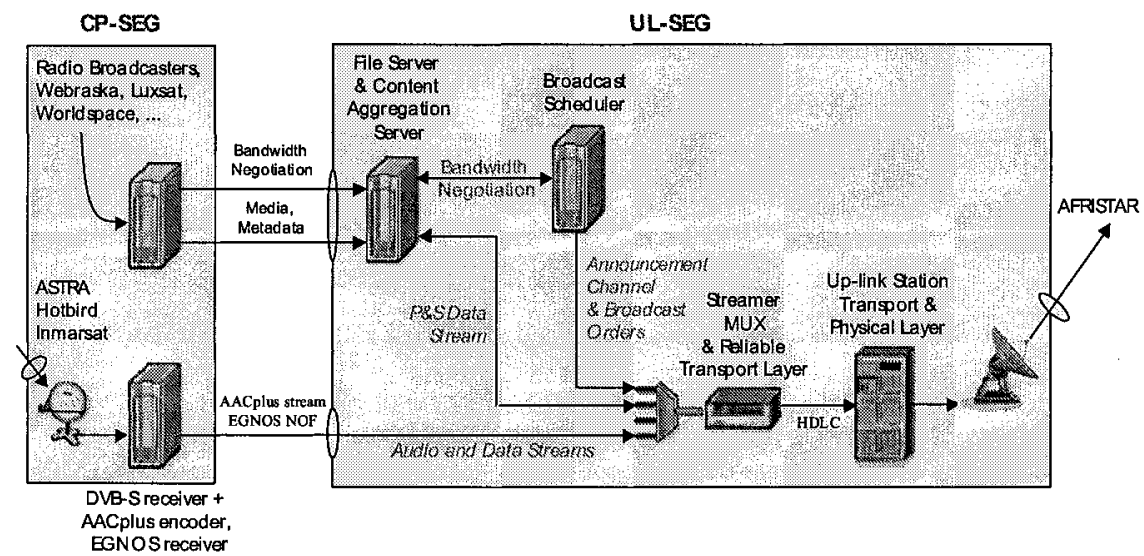

Fig.4 : Content Production and Up-Link Segments

The UL-SEG is composed of two main components :

- The scheduling and content aggregation server : It organises the broadcast of the content aggregated from the different service providers. It applies reliable transport layers protection schemes.

- The up-link station : applies the transport and physical layers to the stream. 
The UL-SEG is interfaced with the CP-SEG and the Space Segment (SPSEG). The UL-SEG constraints are :

- Only $128 \mathrm{kbps}$ will be available for the tests. The useful data rate will be below $64 \mathrm{kbps}$ when time-diversity and application layer encoding are performed.

\subsection{Space Segment}

The Space Segment (SP-SEG) is composed of the AFRISTAR satellite located on a GEO orbit at $21^{\circ} \mathrm{E}$. No specific development on this segment shall be performed in the frame of RELY. The SP-SEG mission is :

- To receive the signal from the up-link station in X-Band,

- To amplify and transmit the signal down to earth in L-Band.

The SP-SEG is interfaced with the UL-SEG, RP-SEG and TR-SEG. The SP-SEG constraints are :

- As AfriStar is designed to cover Africa, it EIRP towards Europe is far below the level that a European system would need (10 to $20 \mathrm{~dB}$ below).

\subsection{Terrestrial Repeaters Segment}

The Terrestrial Repeaters Segment (RP-SEG) is composed of several repeaters located around both the French and German trial sites (respectively in Paris and in Erlangen). The RP-SEG mission is :

- To receive and demodulate the signal from the satellite,

- To modulate and re-transmit the signal with a COFDM waveform (as specified in section 10).

The RP-SEG is interfaced with the SP-SEG and Terminal Segment (TRSEG). The RP-SEG constraints are to obtain a transmission authorisation from the respective regulatory bodies of France and Germany.

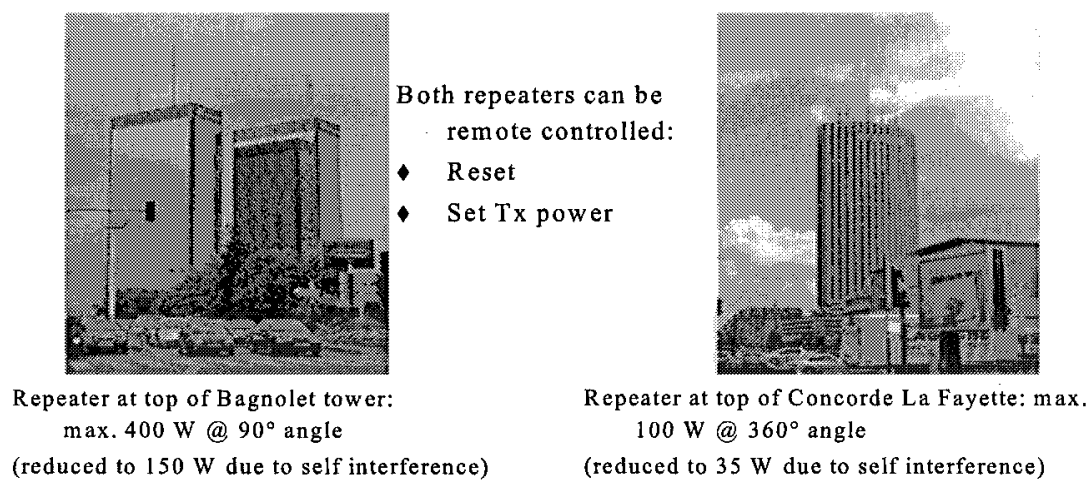

Fig.5 : Repeater segment. Installation of Network in Paris 


\subsection{Terminal Segment}

The Terminal Segment (TR-SEG) is integrated in test and demonstration vehicles. The vehicles is driven in urban and rural environment at both trial sites. The TR-REG mission is :

- To receive and combine the signals received from the satellite and/or the repeaters.

- To decode reliable transport layer schemes.

- To filter the content and cache only the content relevant to the end-user (according to pre-defined user profile).

- Provide the MMI to browse the cached content, access the streamed content, and also to configure the user profile.

- To enable connectivity to the $2 \mathrm{G} / 3 \mathrm{G}$ terrestrial cellular network in order to request additional information or services (like booking an hotel, etc).

- To enable easy synchronisation with handheld devices like pocket PC or Palm pilots with a WIFI interface.

- To estimate an enhanced vehicle location as well as location integrity information thanks to the EGNOS correction data provided by the satellite radio and to a classical GPS receiver.

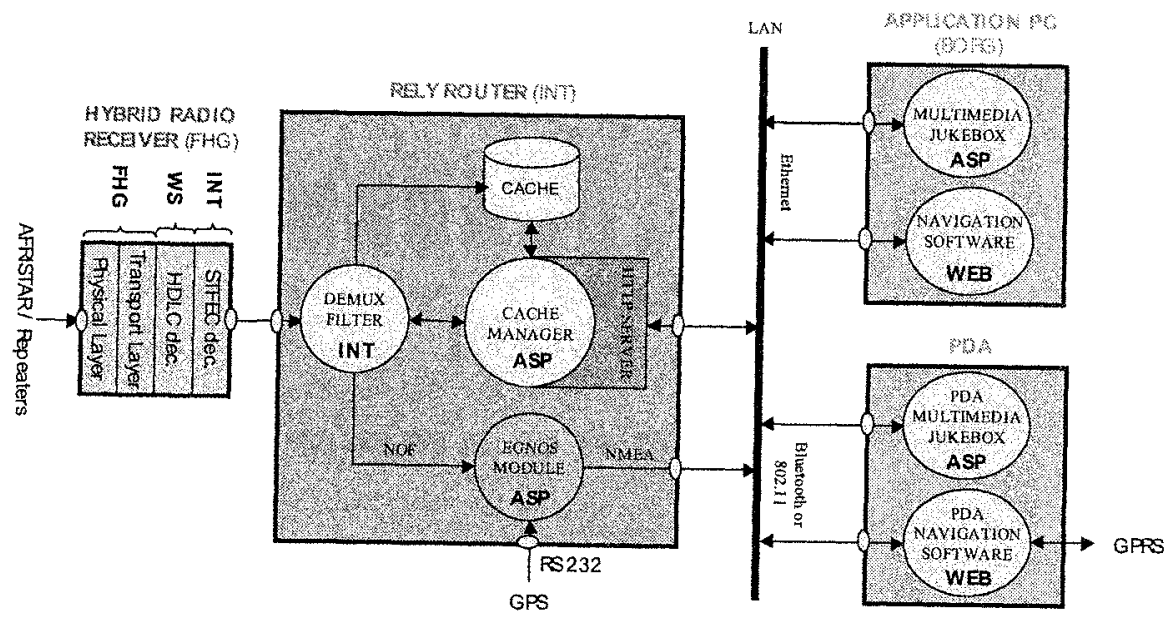

Fig.6 : Terminal Segment Architecture

The TR-SEG is composed of four main physical components :

- The Hybrid Radio Receiver : receives, demodulates and combines the entire satellite and terrestrial TDM' s. The HRR can be considered as a stand-alone car radio, and therefore shall be capable of decoding and playing the received audio streams. The HRR has a data output port. 
- The RELY Router is connected to the "data out" port of the HRR. Its main mission is to enable push-and-store services by implementing intelligent profiling, scheduling the hybrid car radio data reception, and dispatching the received data to the available client applications.

- The EGNOS Module receives GPS raw data from a GPS receiver and the EGNOS data-gram from the RELY Router. It computes enhanced terminal location and location accuracy. In practice the EGNOS Module software will be installed on the RELY Router.

- Application Platforms : The telematics application platforms (embedded console, PDA, Laptop) shall be connected to the RELY Router thanks to a wireless WIFI link or an Ethernet cable.

The TR-SEG is interfaced with the SP-SEG, RP-SEG, with the $2 \mathrm{G} / 3 \mathrm{G}$ network, with additional handheld or portable devices, with the GPS satellites, with the vehicle and with the end-user. TR-REG constraints are :

- The developed solutions on the satellite receiver side and on the EGNOS calculator side will be prototypes and therefore shall not be integrated on a low consuming and small chipset. Consequently, the terminal prototype shall need sufficient room and power supply in the truck of the vehicles (around $80 \mathrm{~W}$ at $12 \mathrm{~V}$ for the satellite receiver prototype).

- The reception of cache content shall be ensured while the engine is off. Therefore, the terminal shall "listen" to the broadcast channel only when necessary, and power saving strategies will have to be implemented.

- The terminal prototype is integrated in vehicles as shown in figure 7:

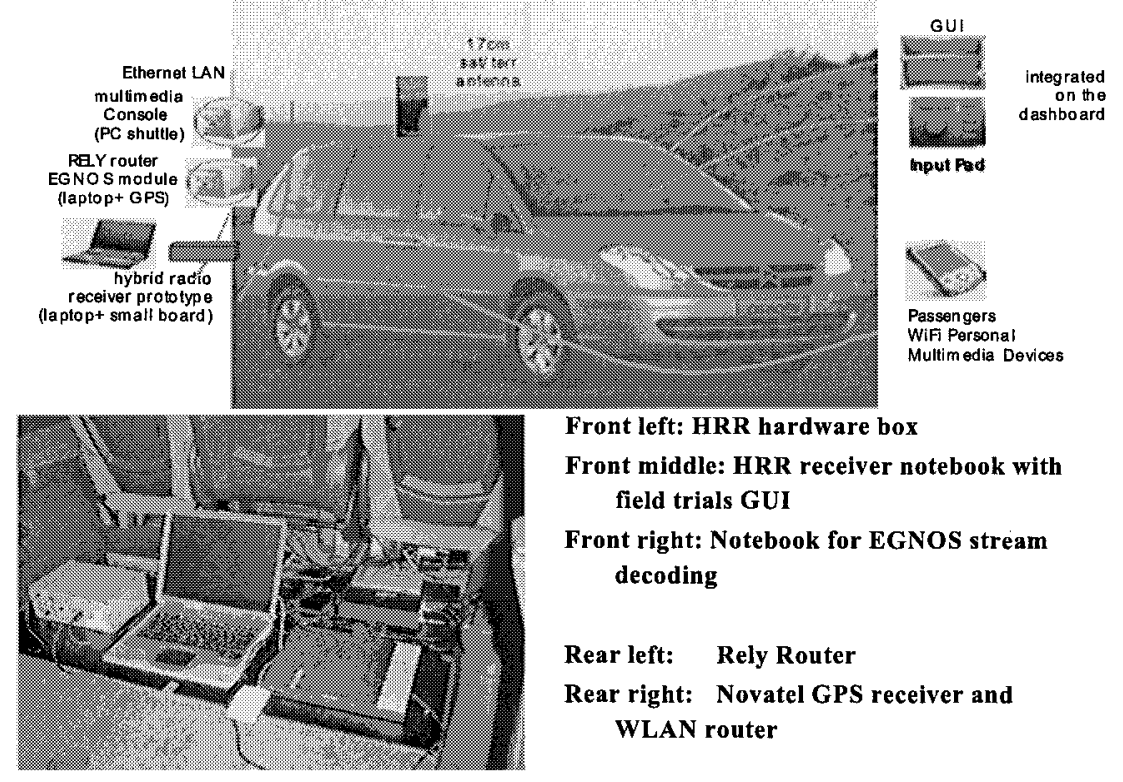

Fig. 7 : Terminal in-vehicle integration 


\section{RELY SYSTEM LAYER ARCHITECTURE}

In order to provide the services defined in section below, RELY follows a layered architecture.

\subsection{Application Layer : Cache Management}

The cache management function consists in filtering a large amount of broadcast data, in order to store only the content corresponding to the user profile in the terminal cache. This method enables to make the best use of the system available bandwidth. This smart caching of content in the cache virtually augments the system bandwidth by providing the user with :

- Content exactly fitted to its tastes or needs,

- The opportunity to enjoy the content "on demand".

In order to make the stored content always fit closer to the user wishes, RELY will implement an adaptive profile management tool. This profile manager will change the user preference settings in function of the user cache usage. For instance, if the user squeezes a given piece of music, it will be interpreted as "the user disliked the content", and the profile manager will decrease the weight of the category to which the content belongs in the user profile.

Almost all the services implemented in RELY will make use of cache management :

- Audio services : The terminal will emulate a so-called "My Station" radio channel with content stored in the cache.

- Navigation services : The terminal will filter geo-referenced data according to the user preference and the terminal location.

- Web-Casting services : Several web-sites will be replicated in the terminal.

- Paging : The paged channel will be stored in the cache if the vehicle belongs to the target fleet.

- ETC : Only ETC vehicles shall receive software upgrades.

\subsection{Reliable Transport Layer}

The transmission of data with a high reliability over a mobile transmission channel requires the use of high layer data protection algorithms. In RELY, such algorithms will be implemented just above the transport layer (Reliable Transport Layer). The reliable transport layers implemented in RELY are of three kinds : 
- Short Term FEC (STFEC) : consists in adding a quite large redundancy to the data $(15-20 \%)$ and to interleave the data over a period of time smaller or equal to a minute.

- Long Term FEC (LTFEC) : consists in adding a small redundancy (around 6\%) and to interleave over a longer period of time (greater than a minute).

- Carrousel or Data Fountaining : consists in sending the same data several times.

\subsection{Security Layer}

The goal of the RELY system security layer is to protect the content of RELY services data in order:

- to control the access to content to authorised users.

- to protect author's rights.

- RELY security layer is entirely driven by a commercial objective:

- services related data are not confidential

- the associated protection aims to generate revenues to RELY system operator by preventing unauthorised access to broadcast services for subscribers.

The security layer is not implemented in RELY testbed.

\subsection{Physical and Transport Layers}

The physical and transport layers implemented in RELY will be highly constrained by the use of the processed payload of the existing AfriStar satellite. RELY will implement a satellite and a terrestrial waveform in order to provide the hybrid system architecture.

\section{RELY SERVICES}

The following service categories have been identified :

- Audio Services

- Web-casting Services

- Navigation Services

- Professional Paging Services

Audio and Web-casting services are gathered in a single application called Radio and Multimedia Jukebox (MJB). The ETC service will be emulated as a subpart of the Web-Casting Service. 


\subsection{Radio and Multimedia juke-box}

Based on a standard car radio interface, the MJB [Multimedia jukebox] will grant access to a wide variety of service :

- Audio services (compatible with the driving activity) : "live" channels and emulated channels.

- Multimedia services (for passengers) : video, web sites replication, etc.

\subsubsection{Audio Services}

RELY will demonstrate audio services as they could be in the future European satellite radio system. These services can be divided into two categories :

- "Live" streaming : the user can listen to audio channels (music or talk) directly off the air. In RELY, the live channels will use the very efficient AACplus audio coding scheme. AACplus enables to reach near $C D$ audio quality at data rates down to $40 \mathrm{kbps}$.

- "Cached" streaming : the user can listen to audio channels generated from the content stored in the cache of its terminal. The principle is the following. The terminal continuously receives audio files from the "live" broadcast channels and from a dedicated high data rate data channel. The audio files are filtered and stored in the terminal cache according to the user profile. This requires to broadcast segmented content and to use an efficient filtering algorithm in the receiver (Remark: In RELY, the capture of content from "live" streams will not be implemented. This would have required a stronger involvement of radio broadcasters. In the future European radio system, it is highly recommended that this feature be implemented.)

The user interface for "live" and "cache" streaming services is similar to that of a standard car radio as shown in the following figures. It must respect the constraints due to the vehicle environment. The user must be able to use the satellite radio while driving.

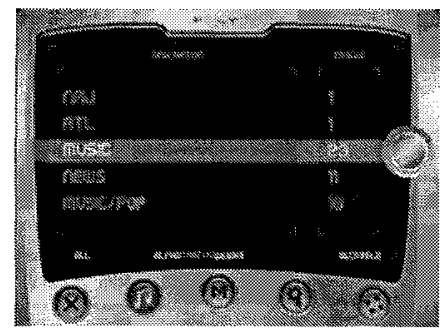

Fig.8 : MJB Radio Service - Channels List 


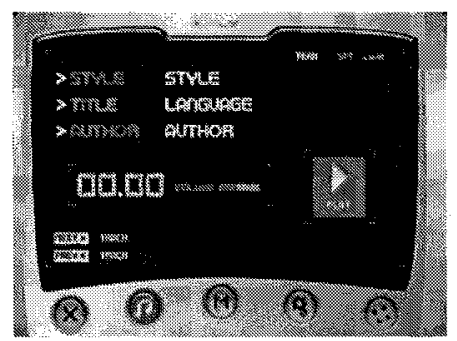

Fig. 9 : Cached Radio GUI

\subsubsection{Web-Casting Services}

\subsubsection{Rich HTML Content}

The web-casting service consists in replicating a given number of websites in the terminal using the efficient satellite digital radio link. The web content is filtered in the terminal in function of the user profile. The user can then browse the content off-line. For safety reasons, this service is mainly targeted to the vehicle passengers. They can access the content either on the car main multimedia console if available (large screen), or on PDA's with WiFi connection to the terminal web server.

\subsection{Navigation Services}

The navigation services demonstrated in RELY can be divided into the following categories:

- Automatic and continuous route optimisation based on real-time traffic information: The driver requests and downloads a corridor of cartographic information over the GSM or GPRS network. Then, while driving, the RELY terminal is able to filter the traffic information received from the radio satellite and to recalculate or get a new itinerary.

- Traffic maps generation: a simplified road network model is already stored in the terminal. The car radio receives vectorised traffic data. The terminal displays this information on the roads network model with colour codes depending on the traffic density.

- Dynamic points of interest notification: This services keeps the driver informed of dynamic parameters changes of pre-defined points of interest (such the number of vacancies in parking), or of any change in normally static parameters (location, new parking).

- Enhanced turn-by-turn navigation: The terminal downloads or calculates an itinerary. The driver is guided along a route with turn-by- 

cellular technology and EGNOS satellite navigation

turn driving instructions triggered by the positioning information provided by the EGNOS-enabled module.

- Vehicle tracking with no-move detection : This service uses the enhanced EGNOS positioning to provide services to professionals such as secured goods transporters, etc.

The RELY navigation services will only run on the car embedded console.
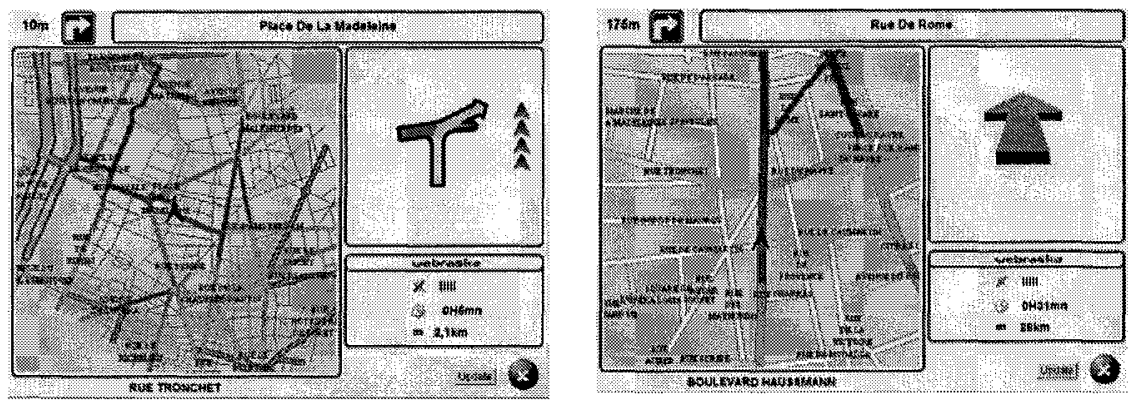

Fig.10: Navigation service

\subsection{Professional Paging Services}

This service consists in multi-casting multimedia short messages to a large fleet of vehicle through the satellite digital radio link. A sound alert warns the driver that a message has been received. These paging services may be of interest for truck companies or car manufacturers. In RELY, Webcasting services provided by the MJB emulates the paging service.

\section{DEMONSTRATIONS}

The main purpose of RELY is to validate and promote new concepts. In order to develop and demonstrate the efficiency of the RELY system in a multi-cultural environment such as Europe, two trial sites are set up, one in Paris (France) and one in Erlangen (Germany). The activities undertaken in the two sites will enable to:

- Validate technologies such as a reliable transport layer, and cache management in mobile environment,

- Make live demonstration of the aforementioned services,

- Assess the benefits of an hybrid broadcast of the EGNOS NOF

- Validate the trans-national operation of the system concept. 
The developed RELY terminals are embedded into vehicles provided by Daimler Chrysler, BMW and PSA. The service applications run on a Borg Instruments platform and on Personal Digital Assistants. The demonstration will be available at the trial sites from June 04 to July 04 .

\section{ACKNOWLEDGEMENTS}

A lot of elements presented in this paper have been produced by the RELY consortium partners, within the frame of the RELY project, funded by the European Commission IST (Information Science Technology).

\section{CONTACTS}

Web Site: http://www.rely-europe.com/

Email: contact@,rely-europe.com 